Mens

revue d'histoire intellectuelle de l'Amérique française

\title{
L'histoire intellectuelle selon Jean de Viguerie
}

\section{Pierre Trépanier}

Volume 2, numéro 1, automne 2001

URI : https://id.erudit.org/iderudit/1024455ar

DOI : https://doi.org/10.7202/1024455ar

Aller au sommaire du numéro

Éditeur(s)

Centre de recherche en civilisation canadienne-française

ISSN

1492-8647 (imprimé)

1927-9299 (numérique)

Découvrir la revue

Citer cet article

Trépanier, P. (2001). L'histoire intellectuelle selon Jean de Viguerie. Mens, 2(1),

5-15. https://doi.org/10.7202/1024455ar d'utilisation que vous pouvez consulter en ligne.

https://apropos.erudit.org/fr/usagers/politique-dutilisation/ 


\title{
PERSPECTIVES
}

\section{L'HISTOIRE INTELLECTUELLE SELON JEAN DE VIGUERIE}

\author{
PIERRE TRÉPANIER \\ Département d'histoire \\ Université de Montréal
}

Les premières fois, je me crus visé pour des raisons politiques, et parce que j'étais classé à droite. Je mis longtemps à réaliser la véritable nature de ma déviation. En fait mon péché n'était pas politique, il était métaphysique. J'étais coupable de dire que l'homme est un être pensant et croyant.

-Jean de Viguerie

«Mais, vous croyez à la nature humaine!» se récrie l'étudiant, d'un air où se disputent la consternation et la pitié. Je débutais dans la carrière. Ce cri du cœur, inattendu et lancé en pleine classe, me désarçonna. Je ne dus pas briller.

Étudiant en anthropologie, il avait bien retenu sa leçon. Les êtres humains sont différents dans l'espace et dans le temps. Comment parler dans ces conditions de nature humaine? De toute façon, ni le structuralisme, ni le marxisme ne se préoccupent des natures. Il n'empêche qu'il faut sacrifier aux droits de l'homme. Ce sont, tranche Le Petit Larousse 2001, les «droits et libertés que chaque individu possède du seul fait de sa nature humaine». Il y a là comme une difficulté logique.

Jean de Viguerie croit à la nature humaine. C'est un historien humaniste, au sens de l'anthropologie chrétienne, pour qui l'homme, tout homme est un corps et une âme spirituelle substantiellement 
unis. Malgré les apparences, cette précision n'est pas un luxe. Elle fonde la théorie du rôle des idées en histoire et conditionne l'exercice du métier d'historien. Par son esprit, l'homme est plus que la matière et que la société; il peut connaître réellement l'une et l'autre; sa pensée est une activité libre et les idées qui en sont le fruit jouissent d'une autonomie au moins relative par rapport aux conditions matérielles. Viguerie est un spécialiste de l'histoire des idées qui prend les idées au sérieux. Ce n'est pas cultiver le paradoxe que de soutenir que beaucoup de spécialistes de l'histoire intellectuelle méprisent les idées. Elles leur paraissent un pur produit de l'économie, des rapports sociaux ou des structures de l'inconscient. On se demande bien pourquoi ils s'entêtent à les étudier et, d'ailleurs, de quel droit ils s'accrochent à leur chaire. Car enfin, si toute pensée n'est qu'une représentation sociale, ce sont des imposteurs et leur science, une mystification. Mais chacun de nous sent au fond de luimême qu'il est plus que l'intersection d'une formation et d'une pratique sociales. Le sentiment de cette dignité peut être d'un grand secours.

Viguerie, qui s'intéresse surtout à l'histoire religieuse et à l'histoire des idées pédagogiques et politiques, ne prétend nullement que l'explication historique, en dernière analyse, est monocausale et qu'il suffit de substituer les idées aux rapports de production. Il a une très vive conscience de la complexité, du foisonnement, de l'épaisseur du passé, objet d'étude de l'historien. Mais il réagit contre «l'histoire structuralo-sociologique, l'histoire blochienne' $»$, sorte d'histoire en l'absence de l'homme qui pense. Car l'homme de cette histoire-là est tout matériel et ne pense pas; il est un nœud de mentalités collectives, traduction sur un autre plan de l'économie et de la société. Viguerie revendique simplement le droit de poser sérieusement «la question redoutée de l'influence des idées sur le cours des événements ${ }^{2} \gg$. Il creuse patiemment jusqu'à ce qu'il atteigne «l'infrastructure philosophique ${ }^{3} »$. Le sachant ou ne le sachant pas, hommes d'action et intellectuels agissent et écrivent dans le cadre d'une philosophie commune, qui est la généralisation, la vulgarisation d'une doctrine philosophique particulière. Tant qu'on n'a pas mis au jour ces bases souterraines, on ne peut prétendre 
connaître les idées d'un groupe ou d'une époque et, par conséquent, saisir adéquatement le rôle historique de ces idées. L'œuvre exemplaire de Viguerie se présente comme l'heureuse démonstration de la fécondité de cette méthode.

La crise de la conscience occidentale trouve à ses yeux une bonne part de son explication dans une série de dissociations mise en branle par le cartésianisme, qui devient la philosophie commune dominante vers 1660. La responsabilité du siècle classique dans la Révolution française et dans la «crise de l'intelligence» apparaît irréfutable. Par son dualisme oublieux de l'unité substantielle de l'homme, Descartes légitimait le mécanisme et même, à son corps défendant, le matérialisme. Mais le triomphe de Descartes ne se comprendrait pas sans l'abandon de la philosophie thomiste, dont les conséquences, insiste Viguerie, sont incalculables ${ }^{4}$. La crise intellectuelle de l'Europe est aussi la crise de l'Église. Or on a assisté au sein de l'Église à un dépérissement de la philosophie traditionnelle, de la scolastique 5 . Dans sa thèse de doctorat sur les Pères de la Doctrine chrétienne ${ }^{6}$, Viguerie montre que si l'on continue à enseigner la philosophie thomiste, on a perdu l'intelligence de ses concepts, auxquels on donne une signification cartésienne ou mécaniste ${ }^{7}$. Les répercussions sur la théologie sont funestes ${ }^{8}$. La foi est entamée; la discipline se relâche; un malaise s'installe. Le clergé, en particulier celui de France, ne peut lutter contre les idées nouvelles, profondément antichrétiennes, pour la raison qu'à son insu il en partage déjà les présupposés.

Dans un essai brillant, Les Deux Patries $^{9}$, Viguerie applique sa méthode aux idées politiques ${ }^{10}$ avec une virtuosité qui éblouit et une maîtrise qui convainc presque toujours. On y voit comment le patriotisme révolutionnaire s'empara du patriotisme ancien, remplaçant sans qu'il y parût la patrie traditionnelle, - la terre des pères, le pays de la naissance et de l'éducation -, par la patrie révolutionnaire, abstraite et utopique. Au lieu de la reconnaissance et de la piété, c'est la démesure, et le sacrifice de millions de vies qu'exige l'insatiable déesse. L'opération de captation est si habilement menée, si parfaitement réussie qu'elle finit par abuser 
tout le monde de sorte que le patriotisme des catholiques, clercs aussi bien que laïcs, et le patriotisme des nationalistes, celui de Maurras comme celui de Barrès, en sont irrémédiablement contaminés. Cela arriva parce que royalistes, catholiques et traditionalistes avaient intériorisé peu à peu le substrat philosophique qui rendait possible la conquête des esprits ". La réaction qu'ils esquissèrent était par conséquent vouée à l'échec. Le point tournant est encore le XVII ${ }^{\mathrm{e}}$ siècle, qui met tout en place pour assurer au siècle suivant la domination des libertins et des philosophes, qui devait aboutir à la Terreur : «l'appauvrissement de l'idée de nature» entraîna «la faiblesse du nouveau concept de la liberté ${ }^{12} »$. Cette domination se reconnaît à ceci qué «nul ne sait plus rien de l'identité de la France, ni de la signification réelle du mot patrie. L'être moral de la France est effacé, le cosmopolitisme triomphe, l'idée de patrie se confond avec celle des droits de l'homme ${ }^{13}$.»

C'est avec une singulière autorité que Viguerie parle du XVIII ${ }^{e}$ siècle. Il a en effet signé l'Histoire et dictionnaire du temps des Lumières ${ }^{14}$. Ne craignant pas de prendre le contre-pied des idées reçues - et l'historiographie en est pleine-, il dévoile les contre-vérités de la philosophie des lumières ainsi que les effets pervers qui ne manquèrent pas d'en sortir et que les révolutionnaires de la Terreur se chargèrent de matérialiser avec une sauvagerie sanglante. Sait-on que le racisme et la xénophobie, que la théorie du sous-homme et celle de la supériorité de l'homme blanc sont inséparables de l'idéologie des Lumières? «Le racisme des Lumières, observe-t-il, est d'origine philosophique. Il vient d'une philosophie matérialiste niant l'existence de l'âme. [...] Il en résulte au moins deux conséquences. La première est que l'on ne voit plus que les différences physiques et que l'on réduit l'humanité à des catégories raciales. La seconde est que l'on confond l'homme et l'animal : "Des animaux à l'homme, écrit La Mettrie, la transition n'est pas violente" (L'Homme-Machine, éd. 1966, p. 78). S'il en est bien ainsi, on ne voit pas pourquoi les animaux ne seraient pas des hommes, les hommes des animaux et les Noirs des singes ${ }^{15}$.» Car ce sont d'abord les Noirs que vise le mépris des «grandes consciences des Lumières». L'historien démonte avec un art consommé la tolérance dont se 
targuent les «philosophes», tolérance limitée et orientée. Pour les Lumières, toute religion constituée est superstition, et toute superstition, signe d'infériorité. Si dans l'interprétation de Viguerie tout n'est pas prouvé, tout est vraisemblable, et il sait distinguer l'hypothèse de la conclusion rigoureusement établie. Témoin ses précisions sur le racisme et l'ethnodifférentialisme ${ }^{16}$.

Viguerie est aussi un spécialiste de l'histoire des idées et de la pratique pédagogiques ${ }^{17}$. Ici encore il perçoit la période $1660-1715$ comme décisive : elle donne naissance à une nouvelle manière d'éduquer les enfants, enracinée dans le cartésianisme et le jansénisme. Comme ils pensent que l'intellect est passif, «les nouveaux pédagogues ne croient plus à la valeur de l'effort intellectuel». La pédagogie devient plus pessimiste ${ }^{18}$. Au XVIII siècle, débute le règne de l'enfant-roi. La discipline fléchit. «Nous connaissons un phénomène analogue, poursuit l'auteur : le déclin de l'observance, c'est-à-dire de la fidélité à la règle, dans la plupart des ordres religieux depuis les dernières décennies du XVII ${ }^{e}$ siècle. Bénédictins, capucins, oratoriens et autres religieux consacrent de moins en moins de temps à la prière, se lèvent de plus en plus tard, sortent en ville sans permission, manquent à la pauvreté ${ }^{19}$.» Dans ses travaux sur l'histoire de l'éducation, l'auteur attache une grande importance à l'enseignement de la philosophie. Pourquoi? «Parce que l'éducation chrétienne est à base d'intelligence. La religion est toujours liée à la philosophie, que cette dernière soit sa servante ou son adversaire ${ }^{20}$.» Il admire l'œuvre éducative de la Réforme catholique : «Comme toutes les pédagogies, la Réforme Catholique a transmis une éthique conforme à sa logique. Fondée sur la réhabilitation des œuvres, elle a formé des chrétiens amis de l'action autant que du savoir, des citoyens pressés de contribuer à l'édification de la cité chrétienne ${ }^{21}$.» L'éducation apparaît comme le baromètre des sociétés et, tôt ou tard, le théâtre de leur philosophie en acte.

Viguerie est non seulement un maître historien, mais aussi un bon écrivain, sobre, efficace, clair. À le lire, l'étudiant se convaincra que l'on peut évoquer les problèmes les plus difficiles dans une langue accessible et mener une démonstration exigeante sans pédantisme. 
En parcourant les études solides de Viguerie, des rapprochements avec l'histoire du Québec se présentent naturellement à l'esprit. Considérons trois problèmes. D'abord la désertion du clergé québécois à l'époque de la Révolution tranquille. Comme me le disait un jour Francois-Albert Angers, ce sont les chefs spirituels qui les premiers ont lâché, et non pas le peuple. On attend encore une explication convaincante du phénomène. Car tous ces frocs, toutes ces soutanes, toutes ces cornettes aux orties, c'est quand même un fait considérable. Je crois que l'historiographie s'en est peu préoccupée parce qu'au fond on approuvait : l'obscurantisme reculait, la raison progressait. L'on est peu porté à s'interroger sur des évidences. Mais le problème reste entier et «l'interprétation sociologico-structuraliste ${ }^{22}{ }$, d'ailleurs plus philosophique qu'historique, n'a pas grand chose à offrir ici. La philosophie dont il s'agit est positiviste, matérialiste et structuraliste; elle nie au religieux sa spécificité. Mais, «depuis 1980, constate l'auteur, on assiste à une sorte d'usure de l'interprétation philosophique ${ }^{23} \gg$. Je propose deux hypothèses, intimement liées et qui toutes deux se rapportent à la formation reçue par le clergé québécois. Mon idée est que ce dernier, dans une proportion élevée de ses membres, s'est trouvé intellectuellement et moralement démuni devant les assauts de la modernité et de l'hédonisme à partir de la fin des années 1950. Il faudrait analyser les manuels, les notes de cours et l'entrainement ascétique dans les grands séminaires. Si la foi traditionnelle s'est révélée si fragile, cela doit tenir en partie à la philosophie et à la théologie enseignées dans les décennies précédentes, et en latin à des étudiants qui possédaient mal cette langue. La difficulté linguistique n'est pas insurmontable; il y a des avantages à étudier la scolastique dans la langue qui, depuis l'origine, lui a servi de véhicule. Le mal était ailleurs. Le thomisme s'était desséché et comme vidé de sa substance. Les vrais maîtres étaient rares. Pour un Louis Lachance ${ }^{24}$, combien de prisonniers du psittacisme? Mais il y a plus. La philosophie médiévale vivait de la controverse; le dialogue et même la polémique lui étaient un aiguillon puissant. Augustinisme, thomisme, scotisme recherchaient la vérité, avec vigueur, passion même, mais du sein du christianisme. Le pluralisme chrétien était la grande idée du philosophe André Dagenais ${ }^{25}$. Or Dagenais n'était 
pas pris au sérieux. Par la faute d'hommes comme le cardinal Villeneuve ${ }^{26}$, l'enseignement thomiste s'est abrité du grand vent de la vie; il s'est assoupi, perdant dangereusement de sa pertinence pour les séminaristes, pour l'illumination de leur raison et l'étaiement de leur foi.

C'est, pour l'homme, union substantielle du corps et de l'âme, s'exposer à de grands périls que de négliger le cœur et la volonté. Or, à la faveur de la prospérité, le clergé québécois s'amollit dans le confort et l'embourgeoisement. Un prophète ${ }^{27} s^{\text {'était }}$ pourtant levé dans les années trente, prêchant aux prêtres le retour au dépouillement évangélique et la nécessité de l'ascétisme. L'archevêque de Québec le fit taire et il eut l'exil en partage. Le clergé québécois n'était pas de taille à affronter la tourmente, ni intellectuellement, ni moralement. Le bon usage de la liberté lui était trop peu familier. Le trouble des esprits et l'émoi des sensibilités étaient grands. Il se laissa gagner par l'entraînement général.

Deuxième problème : l'évolution du nationalisme. Historiens, politologues et sociologues s'en occupent fort. En fait, le nationalisme est mort. La patrie n'est plus un être moral, ni une amitié; la nation n'est plus une solidarité dans le temps; l'histoire vivante s'est réifiée dans le patrimoine. Mais les intellectuels font comme si tout cela vivait encore. Ils veulent seulement civiliser cela, moderniser cela, ouvrir cela à l'Amérique et au monde. La nation culturelle doit céder la place à la citoyenneté, réseau de droits sous l'égide de l'État. La patrie s'efface devant l'État. Et des théoriciens s'autorisent de cette évolution, tout en poussant à la roue, pour justifier le projet souverainiste. Illogisme? Oui, sans doute, s'il s'agissait de la patrie traditionnelle, de la nation culturelle. Mais ces réalités ont vécu. Elles ne peuvent subsister à la déculturation. La Révolution tranquille et paradoxale, qui pour moitié était apparemment une affirmation nationale, a attenté à la mémoire (voyez ce qu'il est advenu de l'enseignement de l'histoire). Elle a ébranlé la culture jusque dans ses profondeurs en la purgeant de la religion. En échange, qu'avait-elle à proposer d'approchant au 
point de vue de l'identité et au point de vue du lien social? En réalité, l'entreprise ne manque pas de logique en ce sens que la patrie qui intéresse les intellectuels n'est pas la patrie des vertus, des valeurs et de la culture, mais la patrie abstraite, celle de l'individualisme et des droits de l'homme. Alors il arrive cette chose extraordinaire que l'on somme les Canadiens français, qui en forment les huit dixièmes, de «se fondre dans l'ensemble québécois ${ }^{28}$ ». Mais ici la logique les rattrape : pourquoi la citoyenneté québécoise plutôt que la canadienne? Comment en est-on venu là?

Troisième problème : l'américanité. C'est la tarte à la crème des historiens d'aujourd'hui. C'est un peu, élargie à tout un continent et mise au goût du jour, une sorte de théorie de la frontière ${ }^{29}$. L'américanité n'est pas la civilisation états-unienne car elle couvre aussi de son aile immense le monde sud-américain. En somme, en Amérique, tout est d'Amérique sauf le Canada français. Il a beau y être enraciné depuis quatre siècles, il n'en est toujours pas. L'américanité lui échappe encore et encore. Pour l'en persuader, on distingue l'élite, indécrottablement francophile, et le peuple, prophète bon enfant de l'américanité la plus pure. - La démonstration de l'américanité populaire est un peu rapide, mais on s'en contentera. - Évidemment, ce résultat intéressant ne s'obtient qu'au prix de quelques simplifications et de quelques oublis opportuns. On avait naguère convaincu cette élite de gallophobie; on l'avait vue se prélasser sans complexe dans le régionalisme ; elle s'oubliait jusqu'à villégiaturer à Old Orchard. Pendant ce temps, aux États-Unis, des universitaires affichaient leur anglophilie; des peintres, des sculpteurs, des écrivains rêvaient d'un pèlerinage à Paris. Qu'importe. Il ne s'agit plus de penser l'histoire; il faut la changer. Les historiens organisent alors une petite déportation morale, l'équivalent de l'amnésie collective du temps de la Révolution tranquille. Mais c'est pour mieux rentrer en américanité. Le réaménagement de l'histoire n'a pas besoin d'autre justification. On rentrera un peu immigrant; en tout cas, beaucoup moins Canadien français; et tellement plus citoyen. Ce sera un charme pour cette majorité renouvelée et rééduquée de se fondre dans la minorité. 
L'une des clés de ces trois problèmes - car toute réalité historique a plusieurs serrures -, est l'idéologie dominante et la philosophie qui la sous-tend. On a longtemps cru que les nationalistes avaient fait la Révolution tranquille et mené le combat de la souveraineté. C'était une illusion. Ce n'est pas pour rien que les traditionalistes ont été exclus de l'une et de l'autre. Leurs auteurs étaient des libéraux. Le libéralisme est un matérialisme, auquel le mécanisme cartésien n'est pas étranger. L'être l'indiffère; l'avoir le comble. Sa théorie de la connaissance est le sensualisme ou l'empirisme, moins étroit; son dogme premier, la propriété; sa morale, l'utilitarisme. Il ne connaît que l'individu et que le temps présent. La recherche du bonheur conçu comme la plus grande somme possible de jouissances évince le bien commun. Il n'aime pas les doctrines. Elles nuisent à la concorde civile. Il préfère les procédures garantes de la paix. On l'a justement appelé procédural. Peu lui importe où l'on s'en va pourvu que la propriété et les aises de l'individu soient sauves. C'est sa définition de la liberté. La patrie est le support temporaire de son projet et sa caution. En fait, il est déjà dans l'aprèspatrie. Le révisionnisme historique devient urgent. Non que l'on révère le passé : il faut tout bonnement occuper le terrain ou, si l'on aime mieux, verrouiller le sens de l'histoire.

\section{NOTES}

1 Jean de Viguerie, Itinéraire d'un historien. Études sur une crise de l'intelligence, XVII'-XVIII' siècle, Bouère (France), Dominique Martin Morin, 2000, 280 p., p. 24. L'épigraphe est tirée de la p. 26.

${ }^{2}$ Ibid., p. 25.

${ }^{3}$ Ibid., p. 278.

${ }^{4}$ Ibid., p. 268.

${ }^{5}$ Ibid., p. 56.

${ }^{6}$ Une ouvre d'éducation sous l'Ancien Régime. Les Pères de la Doctrine chrétienne en France et en Italie (1592-1792), Paris, Nouvelle Aurore, 1976, 702 p. Viguerie était alors maître de conférences à l'université d'Angers. Il est maintenant professeur émérite de l'Université de Lille.

${ }^{7}$ En 1711 , le père Jean-Baptiste Gros enseigne toujours l'hylémorphisme, mais sans prendre parti pour la forme substantielle, 
pourtant indispensable à l'hypothèse. Une æuvre d'éducation, p. 546.

${ }^{8}$ L'auteur signale que vers 1700 de nombreux régents de la Doctrine délaissent la théologie scolastique au profit de la positive, qui «tend à faire de l'Écriture l'unique source de la morale». La théologie positive «ne laisse qu'une petite place à la sagesse humaine, au dynamisme de l'âme et à l'expérience personnelle.» Ibid., p. 625.

${ }^{y}$ Les Deux Patries. Essai historique sur l'idée de patrie en France, Bouère (France), Dominique Martin Morin, 1998, 279 p.

${ }^{16}$ Il se révèle un excellent historien du politique. Sa mise au point sur la révocation de l'édit de Nantes est un chef-d'œuvre, que l'on devrait offrir à l'imitation des apprentis historiens. Ibid., p. 112-139.

11 «On peut s'étonner de voir les patriotismes de droite et de gauche présenter les mêmes caractères révolutionnaires, mais si les philosophies sont les mêmes, il n'y a pas lieu d'en être surpris.» Ibid, p. 163.

12 Ibid., p. 108-109.

13 Ibid., p. 74.

${ }^{14}$ Histoire et dictionnaire du temps des Lumières, Paris, Robert Laffont, 1995, 1730 p. L'œuvre de Viguerie impressionne : une dizaine de livres et une centaine d'articles. Elle porte principalement sur les XVII' et XVIII' siècles.

15 Ibid., p. 1319.

${ }^{16}$ Itinéraire, op. cit., p. 45.

${ }^{17}$ Outre Une æuvre d'éducation, on lui doit l'Institution des enfants. L'éducation en France, XVI'-XVIII' siècle, Paris, Calmann-Lévy, $1978,330 \mathrm{p}$.

${ }^{18}$ L'Institution des enfants, op. cit., p. 312-313; Une cuvre d'éducation, op. cit., p. 626.

19 L'Institution des enfants, op.cit., p. 251.

20) Une auvre d'éducation, op. cit., p. [4].

${ }^{21}$ Ibid., p. 631.

22 Itinéraire, op. cit., p. 279.

2.3 Ibid., p. 280.

${ }^{24}$ Louis Lachance, o.p. (1899-1963). Dominicain et philosophe. Voir Yves Bégin, «Une lettre de Louis Lachance, o.p., à Lionel Groulx [s.d.]» (édition critique), dans Mens, vol. 1, no. 2 (printemps 2001), pp. 163-169.

${ }^{25}$ Journaliste et professeur de philosophie né en 1917.

${ }^{26}$ Quel triste rôle joué en 1927 par le père Villeneuve! Voir Autour 
de Duns Scot. Polémique, présentation de Michel Brindamour, [s.l.], Éditions du Beffroi, 1989,69 p.

${ }^{27}$ Onésime Lacouture (1881-1951). Prédicateur jésuite.

${ }^{28}$ Nikolas Ducharme, «Les trois piliers de la citoyenneté québécoise», Le Devoir, 24 août 2001, p. A8.

${ }^{29}$ Frederick Jackson Turner, «The Significance of the Frontier in American History», 1893. 